\title{
Photoluminescence of $p$-Doped Quantum Wells with Strong Spin Splitting
}

\author{
H. Boukari ${ }^{a, *}$, P. Kossacki ${ }^{a, b, c}$, M. Bertolini ${ }^{a}$, D. Ferrand ${ }^{a}$, \\ J. Cibert ${ }^{a, d}$, S. Tatarenko ${ }^{a}$, J.A. Gaj $^{b}$, B. Deveaud ${ }^{c}$ \\ AND V. CIULIN ${ }^{c}$ \\ ${ }^{a}$ Nanophysique et Semiconducteurs group \\ CEA-CNRS-Université J. Fourier Grenoble \\ Laboratoire de Spectrométrie Physique \\ B.P. 87, 38402 St Martin d'Hères cedex, France \\ ${ }^{b}$ Institute of Experimental Physics, Warsaw University \\ Hoża 69, 00-681 Warszawa, Poland \\ ${ }^{c}$ Institute of Quantum Electronics and Photonics
}

Ecole Polytechnique Fédérale de Lausanne (EPFL), 1015 Lausanne, Switzerland ${ }^{d}$ Laboratoire Louis Néel, CNRS, B.P. 166, 38042 Grenoble cedex 9, France

Photoluminescence of $p$-type modulation doped (Cd,Mn)Te quantum wells is studied with carrier density up to $5 \times 10^{11} \mathrm{~cm}^{-2}$ at various spin splittings. This splitting can be made larger than the characteristic energies of the system thanks to the giant Zeeman effect. At small spin splitting and regardless of the carrier density, the photoluminescence exhibits a single line, which corresponds to the charged exciton in the singlet state. Above a certain spin splitting, the charged exciton is destabilized in favor of the exciton at vanishing hole density, and in favor of a double line at higher carrier density. It is found here that the charged exciton destabilization energy hardly depends on the carrier density. The double line is found to be band-to-band like, with the same initial state - where the holes have the same spin orientation - and final states that differ by some excitation of the $2 \mathrm{D}$ hole gas. In addition, the spin splitting needed to fully polarize the hole gas is twice smaller than expected from the single particle image and gives a unique insight into many-body effects in the hole gas.

PACS numbers: 71.35.-y, 71.55.Gs, 73.21.Fg, 75.30.Hx, 75.50.Pp

*Present address: IMEC, MCP/ART, Kapeldreeef 75, 3001 Leuven, Belgium. 


\section{Introduction}

Investigation of positively $\left(\mathrm{X}^{+}\right)$and negatively $\left(\mathrm{X}^{-}\right)$charged excitons, predicted almost 50 years ago [1], has become possible thanks to the progress achieved in nanostructure technology during recent decades. It is 10 years ago since a charged exciton was observed for the first time in CdTe based quantum well [2] followed by many other studies dedicated to the physics of charged excitons in quantum wells (QWs). In the low carrier density regime, binding energies have been measured in different materials [3-7], while selection rules [8-10] and dynamics [11-16] offer useful tools for the identification of the complexes. The spin configuration of the charged excitons usually corresponds to the singlet state the two identical particles have opposite spins. However, it has been shown that the triplet configuration (the two identical particles having the same spin) can be stabilized in high magnetic field [17-21] thanks to the "shrinking" of the wave functions.

When the free carrier density is increased, the situation is less well understood. For example, it has been reported by several groups that the splitting between the exciton $(\mathrm{X})$ and charged exciton absorption lines increases [3, 22-24] with the free carrier density, while the intensity of $\mathrm{X}$ decreases in favor of the charged exciton, which finally turns into the Fermi edge singularity [25, 26]. However, it has been shown theoretically that the splitting of the absorption lines is not a direct measure of the binding energy [27, 28].

Here we focus on photoluminescence (PL), observed in modulation $p$-type doped QWs at various spin splittings, which can be made larger than some characteristic energies of the system before entering the Landau level regime. This is possible thanks to the well-known giant Zeeman effect in (Cd,Mn)Te. We show that a detailed analysis of the states involved in the PL process gives access to a variety of information such as the enhancement of the hole gas polarizability - due to many-body effects - the dissociation energy of the singlet $\mathrm{X}^{+}$which hardly depends on the hole density, and certain excitations of the gas that have to be taken into account in order to describe the final states of the PL.

This paper is organized as follows. Section 2 describes the samples and the experimental setup. Section 3 describes a useful situation in the presence of a low carrier density: the destabilization of $\mathrm{X}^{+}$in favor of $\mathrm{PL}$ from $\mathrm{X}$ as the magnetic field increases. Section 4 corresponds to the core content of this paper. It describes a possible mechanism responsible for the destabilization of the $\mathrm{X}^{+}$in favor of a double line ( $D_{\text {low }}$ and $D_{\text {high }}$ ), which is band-to-band like. Particular attention is given to the determination of the initial and final states involved in the PL

processes. Finally, Sec. 5 presents the characteristic energies that are accessible by optical spectroscopy and compares them to the Fermi energy of the hole gas. 


\section{Samples and setup}

All the samples were grown by molecular beam epitaxy on (001) oriented $\mathrm{Cd}_{0.88} \mathrm{Zn}_{0.12}$ Te substrates. The $\mathrm{Cd}_{0.65} \mathrm{Zn}_{0.08} M g_{0.27} \mathrm{Te}$ barriers allow a pseudomorphical growth as well as a good confinement of the carriers in the 8 or $10 \mathrm{~nm}$ $\mathrm{Cd}_{1-x} \mathrm{Mn}_{x} \mathrm{Te} \mathrm{QW}$ ( $x$ varying from 0.0007 to 0.05 ). Because of strain and confinement, the heavy-hole is the ground state of the valence band, splitted by 30 to $40 \mathrm{meV}$ from the light hole. All the energies that will be encountered in the following, for example the Fermi energy of the hole gas, are a few meV at maximum, so that only the first heavy hole state will be involved in the optical transitions discussed here.

Most of the samples were modulation doped by introduction of nitrogen in the barriers. Others were naturally $p$-type doped due to the formation of acceptor states at the surface of the cap-layer [29]. The hole density could be decreased from the $10^{11} \mathrm{~cm}^{-2}$ range to the low $10^{10} \mathrm{~cm}^{-2}$ range by above barrier illumination $[24,30,31]$, using an Ar-ion laser. We also tuned the hole density from the low $10^{10} \mathrm{~cm}^{-2}$ to $4 \times 10^{11} \mathrm{~cm}^{-2}$ by applying bias voltage of the order of $1 \mathrm{~V}$ in some pin structures containing a $(\mathrm{Cd}, \mathrm{Mn}) \mathrm{Te} \mathrm{QW}$ [32]. The hole densities were calibrated from the energy difference between absorption and PL (the so-called Moss-Burstein shift), from the Hall effect or from the filling factors measured at high magnetic fields [33].

The samples were mounted strain-free in liquid He and the magneto-optical measurements were performed in the Faraday configuration. PL was excited with either a tunable Ti-sapphire laser or a HeNe laser, with power reduced to about $2 \mathrm{~mW} \mathrm{~cm}{ }^{-2}$ by placing optical density filters in the excitation beam. Light was collected by a 2D Si-based CCD or a photomultiplier after dispersion by a monochromator. Time resolved PL was excited with a picosecond, tunable Ti-sapphire laser with a $80 \mathrm{MHz}$ repetition rate, a pulse width of $2 \mathrm{ps}$ and an average power less than $100 \mathrm{~mW} \mathrm{~cm}{ }^{-2}$. The $2 \mathrm{D}$ streak camera mounted at the output of the spectrometer had a resolution better than 10 ps.

\section{PL at low carrier concentration}

Typical behavior of the PL as a function of magnetic field in the low hole density regime is illustrated in Fig. 1 for a $8 \mathrm{~nm} \mathrm{Cd}_{0.9963} \mathrm{Mn}_{0.0037} \mathrm{Te} \mathrm{QW}$ almost completely depleted of holes by the Ar-ion laser beam used for the excitation. At zero magnetic field, we observe (Fig. 1a) a strong peak at $1635 \mathrm{meV}$ and a weak shoulder at around $1638 \mathrm{meV}$ which we attribute to the $\mathrm{X}^{+}$and the $\mathrm{X}$ respectively. On increasing the magnetic field, in $\sigma^{-}$polarization (dotted line in Fig. 1a) we observe that the $\mathrm{X}^{+}$signal shifts due to the giant Zeeman effect and its intensity falls to zero by $1 \mathrm{~T}$ (the $\mathrm{X}$ is already gone at $0.25 \mathrm{~T}$ ). In $\sigma^{+}$polarization, we observe a decrease in the $\mathrm{X}^{+}$peak intensity in favor of $\mathrm{X}$ as shown in Fig. 1b. From the latter graph, it is possible to determine the magnetic field at which $\mathrm{X}^{+}$ 

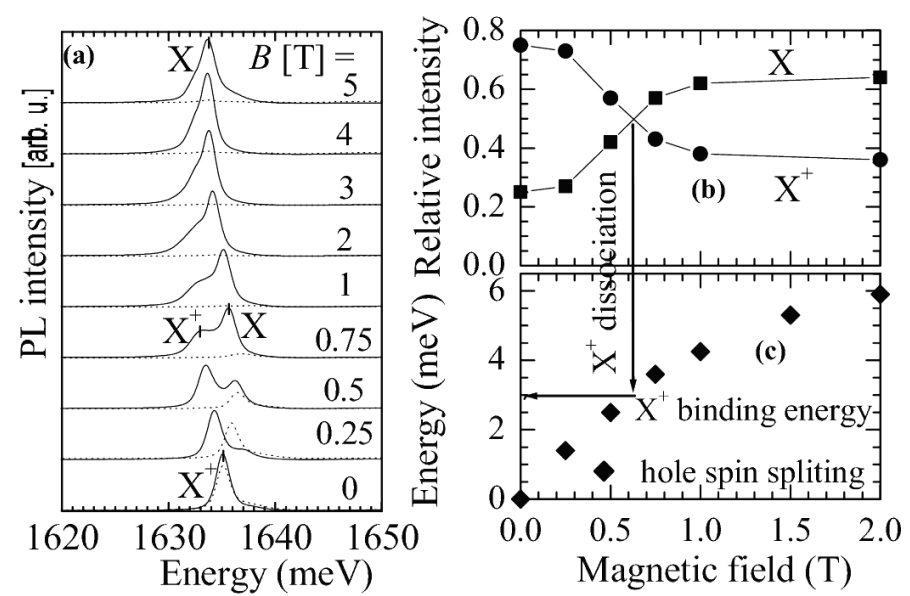

Fig. 1. (a) Photoluminescence spectra acquired at various magnetic fields at $1.7 \mathrm{~K}$, for a $8 \mathrm{~nm} \mathrm{Cd}_{0.9963} \mathrm{Mn}_{0.0037} \mathrm{Te} \mathrm{QW}$ almost completely depleted in holes by the Ar-ion laser beam used for the excitation. The solid traces correspond to $\sigma^{+}$polarization and the dotted traces to $\sigma^{-}$polarization. (b) Relative intensities of the $\mathrm{X}$ and $\mathrm{X}^{+}$observed in $\sigma^{+}$polarization in (a). (c) Valence band spin splitting (diamonds) compared to the binding energy of $\mathrm{X}^{+}$, defined as the difference between the $\mathrm{X}^{+}$and $\mathrm{X}$ energy. The field at which $\mathrm{X}^{+}$dissociation occurs is marked by the vertical arrow.

dissociation occurs. A comparison with the valence hole splitting (Fig. 1c) shows that the destabilization of $\mathrm{X}^{+}$takes place when the hole spin splitting equals the binding energy of $\mathrm{X}^{+}$.

The destabilization of $\mathrm{X}^{+}$in favor of $\mathrm{X}$ can be understood from the different Zeeman splittings involved in a three-particle scheme as shown in Fig. 2. Let us consider one hole initially present in the QW and take as an energy reference the $+3 / 2(\Uparrow)$ hole state. Applying a magnetic field leads to an increase in the energy of the $-3 / 2(\Downarrow)$ hole due to the splitting of the valence band $\left(2 E_{z}^{\mathrm{v}}\right)$; this is the energy needed to flip one hole spin. Absorption of a photon may lead to the formation of $\mathrm{X}$ or singlet $\mathrm{X}^{+}$, separated by the binding energy. Since the two holes involved in the $\mathrm{X}^{+}$have antiparallel spins, the energy states of the $\mathrm{X}^{+}$evolve as the Zeeman shift of the electron $\left(E_{z}^{\mathrm{c}}\right)$. This leads to a shift $E_{z}^{\mathrm{v}} \pm E_{z}^{\mathrm{c}}$ respectively for electron spin $+1 / 2(\uparrow)$ and $-1 / 2(\downarrow)$, with respect to the chosen ground state ( $\Uparrow$ hole). One should also note that the $\mathrm{X}^{+} \mathrm{PL}$ in $\sigma^{-}$polarization leaves the system in its ground state whereas in $\sigma^{+}$it leaves it with a hole in the minority band: a spin flip has to occur in order to reach the ground state.

The exciton X may emit in $\sigma^{+}$polarization when it involves a $\uparrow$ electron and $\Downarrow$ hole or in $\sigma^{-}$when a $\downarrow$ electron recombines with a $\Uparrow$ hole. The second hole (unbound) can take two possible spin orientation so that 4 states involving the bright $\mathrm{X}$ and an unbound hole are defined. We focus on the one where both holes are in the majority spin band $(\Uparrow)$. The Zeeman energy of the latter state shifts as 


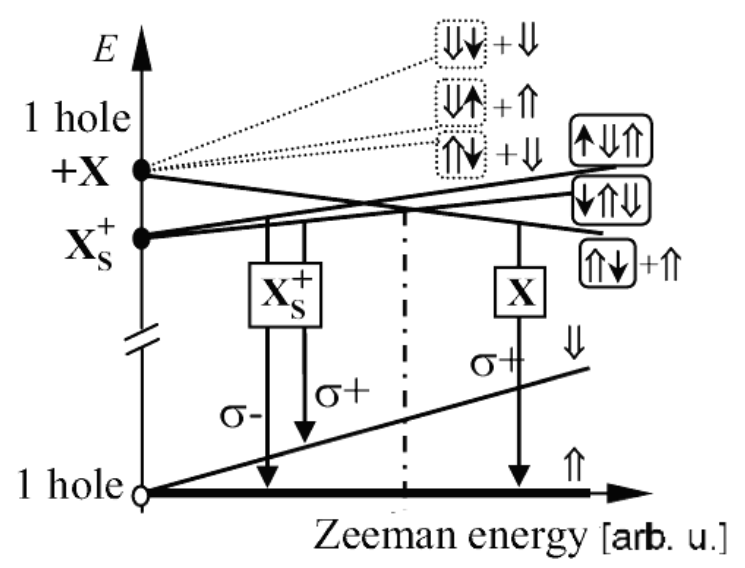

Fig. 2. Zeeman shift diagram of the three-particle initial and final states involved in the system: the solid lines are states that play a role in the PL process while the dotted lines are states represented for completeness. Double line arrows represent the hole spin orientation and the thin single line arrows - the electron spin.

$-E_{z}^{\mathrm{v}}+E_{z}^{\mathrm{c}}$ so that it crosses $\mathrm{X}^{+}$when the hole spin splitting equals the binding energy of $\mathrm{X}^{+}$. We have checked, using samples with different Mn content, that the relevant quantity is the spin splitting and not the applied magnetic field.

To conclude this section, the hole Zeeman splitting can be made large enough to destabilize singlet $\mathrm{X}^{+}$in favor of $\mathrm{X}$ emitting in $\sigma^{+}$polarization. This kind of mechanism has already been discussed in bulk diluted magnetic semiconductors by Planel et al. [34]: there, the exciton bound to a donor $\left(\mathrm{A}^{0} \mathrm{X}\right)$ can be destabilized in favor of the free exciton $\mathrm{X}$ when the hole spin splitting becomes greater than the binding energy of $\mathrm{A}^{0} \mathrm{X}$.

\section{Photoluminescence in the presence of a $2 \mathrm{D}$ hole gas}

PL spectra in the presence of a larger hole density exhibit more complex features as illustrated in Fig. 3 for a $8 \mathrm{~nm} \mathrm{Cd}_{0.996} \mathrm{Mn}_{0.004} \mathrm{Te} \mathrm{QW}$ with a hole density $p=3.2 \times 10^{11} \mathrm{~cm}^{-2}$. At small magnetic field, only one line is observable in both polarizations. Based on our previous experience in $\mathrm{X}^{+}$spectroscopy we ascribe this line to singlet $\mathrm{X}^{+}[24,33]$. When increasing the magnetic field, the intensity of this line decreases in both polarizations: the signal is zero above $0.875 \mathrm{~T}$ in $\sigma^{-}$whereas a novel feature - a double line called $D_{\text {high }}$ and $D_{\text {low }}$ in the following - emerges in $\sigma^{+}$polarization.

Our description of the PL in the presence of a hole gas is based on the scheme illustrated in Fig. 4, where we assume the field dependence of the involved states is as presented in Sec. 3. The hole gas is treated as a surrounding sea responsible for the $k$-space filling. 


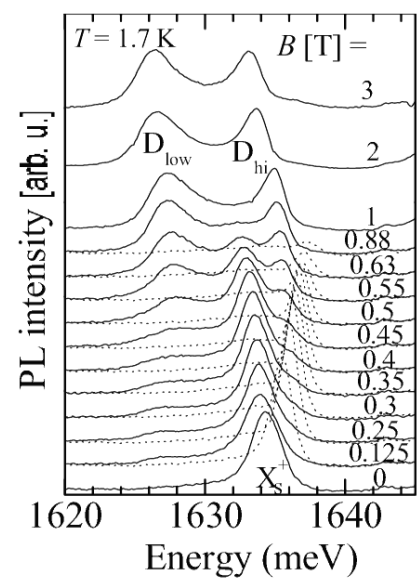

Fig. 3. Photoluminescence spectra acquired at different magnetic fields at $1.7 \mathrm{~K}$ for a $8 \mathrm{~nm} \mathrm{Cd} \mathrm{Cd}_{096} \mathrm{Mn}_{0.004} \mathrm{Te} \mathrm{QW}$ with hole population $p=3.2 \times 10^{11} \mathrm{~cm}^{-2}$. The solid traces correspond to $\sigma^{+}$polarization and the dotted traces to $\sigma^{-}$polarization.

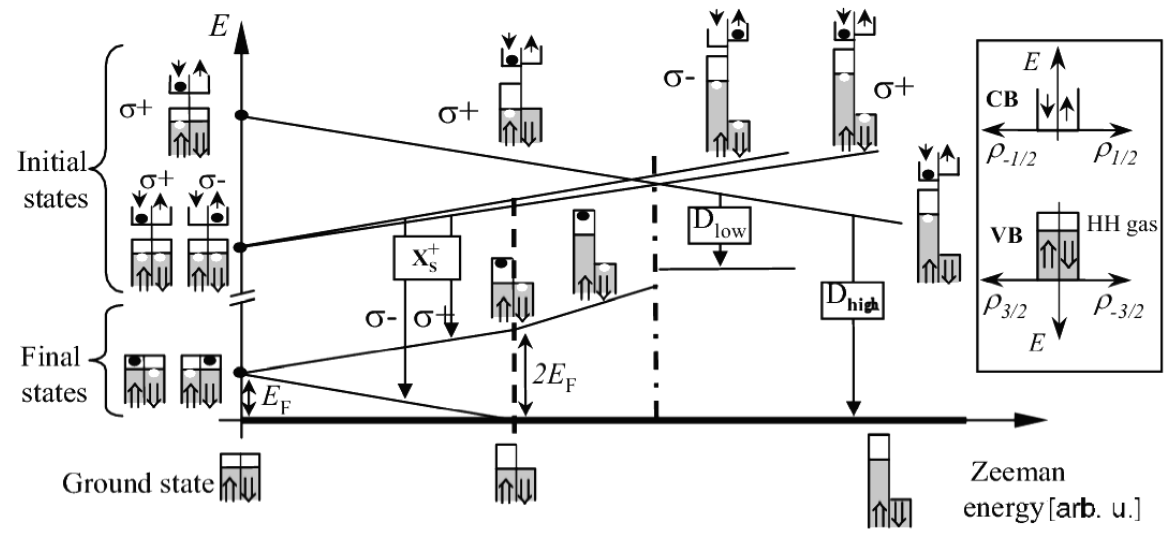

Fig. 4. Representation of the Zeeman shift of the initial and final states involved in the PL process in the presence of a hole gas. White circles correspond to holes and black circles to electrons. The gas is treated as a $k$-space filling sea and illustrated in a energy-density of states picture described in the inset where $\rho$ describes the density of states for the electron spin configuration $\left(\rho_{-1 / 2}\right.$ and $\rho_{1 / 2}$ respectively for the $-1 / 2(\downarrow)$ and $1 / 2(\uparrow)$ electron spin density of states) and $\rho_{-3 / 2}$ and $\rho_{3 / 2}$ correspond to the $-3 / 2(\Downarrow)$ and $3 / 2(\Uparrow)$ hole density of states.

\section{1. $D_{\text {low }}$ and $D_{\text {high }}$ description}

\subsubsection{The same initial state which crosses $X^{+}$}

The initial states responsible for the $D_{\text {low }}$ and $D_{\text {high }}$ transitions were first investigated by time resolved spectroscopy. The magnetic field was $0.5 \mathrm{~T}$, which 
makes it possible to observe $\mathrm{X}^{+}, D_{\text {low }}$, and $D_{\text {high }}$ together (see Fig. 3 ). The excitation laser beam and the detection were both $\sigma^{+}$polarized. The rise time of $D_{\text {low }}$ and $D_{\text {high }}$ were found to be the same, about $2 \mathrm{ps}$, and faster than the $\mathrm{X}^{+}$ rise time, which was larger than 3 ps. When increasing the magnetic field, the rise time of $D_{\text {low }}$ and $D_{\text {high }}$ remain the same and very fast. The fact that $D_{\text {low }}$ and $D_{\text {high }}$ have the same rise time suggests that both transitions arise from the same initial state. Furthermore, the $\sigma^{+}$excitation creates a $\downarrow$ electron and $\Uparrow$ hole so that a hole spin flip is needed to form a singlet $\mathrm{X}^{+}$. The heavy-hole spin flip time measured in $\mathrm{X}^{-}[16,35]$ compares well with the $3 \mathrm{ps}$ measured here. The faster rise time for $D_{\text {low }}$ and $D_{\text {high }}$ suggests also that no spin flip is needed for the formation of the initial state.

The mechanism of destabilization of singlet $\mathrm{X}^{+}$by crossing with another state where the holes are all in the majority band - illustrated in Fig. 4 - is also supported by the evolution of the decay times. It is about $80 \mathrm{ps}$ at zero magnetic field and about $140 \mathrm{ps}$ at $3 \mathrm{~T}$. The fact that the double line is associated with a slower decay channel is in good agreement with the suggestion of having the initial state of $D_{\text {low }}$ and $D_{\text {high }}$ being less correlated than the $\mathrm{X}^{+}$one. The evolution of the decay time is an average of the decay times at $0 \mathrm{~T}$ and $3 \mathrm{~T}$ weighted by the relative intensities of the two PL components (one for $\mathrm{X}^{+}$and the second for $D_{\text {low }}$ and $D_{\text {high }}$ ), which confirms the oscillator strength transfer from $\mathrm{X}^{+}$to the double structure $\left(D_{\text {low }}, D_{\text {high }}\right)$.

As a conclusion, $D_{\text {low }}$ and $D_{\text {high }}$ arise from the same initial state - where the holes have the same spin orientation - and involve less correlated particles than singlet $\mathrm{X}^{+}$.

\subsubsection{Final states of $D_{\text {low }}$ and $D_{\text {high }}$ differ by some excitation of the hole gas}

The determination of the final state of transitions $D_{\text {low }}$ and $D_{\text {high }}$ is made at the crossing point of their initial state with singlet $\mathrm{X}^{+}$. At this particular spin splitting (see Fig. 4), the initial states of all transitions observed in $\sigma^{+}$polarization are degenerate and differ from singlet $\mathrm{X}^{+}$emitting in $\sigma^{-}$polarization by the electron spin splitting. As already mentioned in Sec. 3, the $\mathrm{X}^{+}$emitting in $\sigma^{-}$ polarization leads to the ground state of the system. From the comparison between the position of $D_{\text {high }}$ and $\mathrm{X}^{+}$(see Fig. 3) we conclude that the final state of $D_{\text {high }}$ is also the ground state. Indeed, their energy difference is about $1 \mathrm{meV}: 0.5 \mathrm{meV}$ arises from the electron spin splitting and there is some uncertainty in the position due to the line decomposition procedure.

Therefore, $D_{\text {high }}$ drives one electron from the bottom of the conduction band to the Fermi level of the fully polarized hole gas. This is an indirect transition in $k$-space, normally forbidden. We see large changes in the relative intensities of $D_{\text {high }}$ and $D_{\text {low }}$ from sample to sample, suggesting that the indirect character is allowed by disorder or many-body interactions. Further confirmation of the 
indirect character of the transition is given by the LO-phonon replica that we observe for all samples in $\sigma^{+}$polarization, and only for $D_{\text {high [33] }}$.

The difference between $D_{\text {high }}$ and $D_{\text {low }}$ is then a measure of the energy that is left in the system during the PL process $D_{\text {low }}$ : the final state of the latter transition is an excited state of the hole gas. We will come back to these excitations, compared to the Fermi energy in Sec. 5.

Finally, we keep in mind that $D_{\text {high }}$ is an indirect transition leading to the ground state of the hole gas while $D_{\text {low }}$ is foreseen as being direct in $k$-space so that an electron from the bottom of the conduction band may recombine with a hole at the top of the valence band.

\subsection{Polarization of the hole gas at small spin splitting}

We now turn to the situation of small spin splitting, where the hole gas begins to polarize. Figure 5 shows the positions of the PL and absorption peaks observed

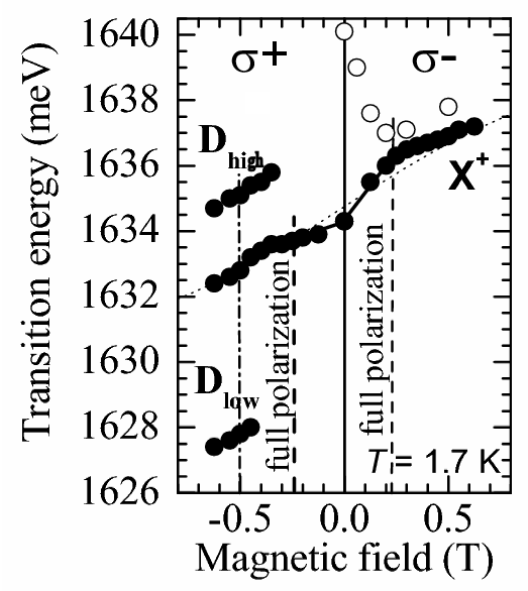

Fig. 5. Energy positions of the PL (full circles) and transmission from the $8 \mathrm{~nm}$ $\mathrm{Cd}_{0.996} \mathrm{Mn}_{0.004} \mathrm{Te} \mathrm{QW}$ with a hole population $p=3.2 \times 10^{11} \mathrm{~cm}^{-2}$. The vertical dashed lines show the field that corresponds to full hole gas polarization and the dash-dotted line mark the destabilization field of $\mathrm{X}^{+}$.

from the $8 \mathrm{~nm} \mathrm{Cd}_{0.996} \mathrm{Mn}_{0.004} \mathrm{Te} \mathrm{QW}$ with a hole density $p=3.2 \times 10^{11} \mathrm{~cm}^{-2}$. In $\sigma^{-}$polarization, we observe a decrease in the difference between absorption and PL positions while increasing the magnetic field to a certain value, which corresponds to the full polarization of the hole gas. This gives a measure of the magnetic field, and thus of the hole spin splitting, needed to fully polarize the hole gas. A comparison of this spin splitting with the Fermi energy of the hole gas will be given in Sec. 5 .

Another remarkable feature is observable in Fig. 6. The positions of the PL peaks do not reproduce the expected modified Brillouin function (dotted line in 


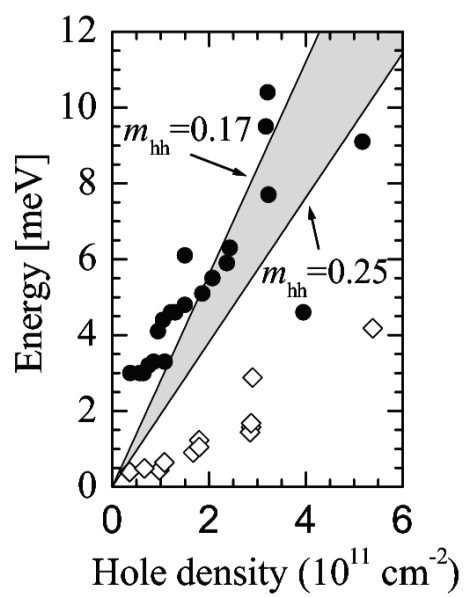

Fig. 6. Spin splitting of the valence band at the limit of full hole gas polarization (open diamonds) and excitation left in the system after the $D_{\text {low }}$ transition give by the $D_{\text {low }}-D_{\text {high }}$ difference (filled circles).

Fig. 6) when the hole gas is only partially polarized. To understand this point, let us return to Fig. 4. At zero magnetic field, the recombination of $\mathrm{X}^{+}$in both polarizations leads to a final state with an electron at the top of the valence band: a spin-flip has to occur and the electron may lose (neglecting many-body effects) an energy equal to the Fermi energy of the gas, $E_{\mathrm{F}}$, in order to reach the ground state. At the limit of a fully polarized hole gas (vertical dotted line in Fig. 4), the final state in the $\sigma^{-}$polarization is the ground state whereas in $\sigma^{+}$polarization an electron is left at the top of the minority hole spin band. The electron has an excess energy corresponding to the Fermi energy at full polarization, $2 E_{\mathrm{F}}$. When the magnetic field is increased to the limiting value where the gas is fully polarized, the excess energy of the final state decreases, from $E_{\mathrm{F}}$ to 0 after recombination in $\sigma^{-}$polarization and increases from $E_{\mathrm{F}}$ to $2 E_{\mathrm{F}}$ after recombination in $\sigma^{+}$polarization. The slopes of the final states are given by the Zeeman shift of the valence band. Thus, the Zeeman shifts of the initial and final states compensate in $\sigma^{+}$ polarization, reducing the shift of the PL peak position, while they add in $\sigma^{-}$ polarization so that the shift of the PL peak position is enhanced - as observed in Fig. 6.

\section{Characteristic energies involved in the system}

The analysis presented up to here gives us the opportunity to extract three characteristic energies of the system. The first one corresponds to the hole spin splitting needed to fully polarize the hole gas. The single particle picture cannot explain the observed spin splitting at full polarization (open symbols in Fig. 6) which is twice smaller than the expected Fermi energy. The latter quantity is 
calculated from the hole density $p$, and the hole effective mass $m^{*}$ which depends on the description of the valence band: $m^{*}=0.25 m_{0}$ in Ref. [36] and $m^{*}=0.17 m_{0}$ in Ref. [37]. Increase in the polarizability of an electron gas, attributed to many-body effects, has already been observed [38-41]. Our system gives a unique insight into many-body effects in a hole gas, where further theoretical work has to be done in order to achieve a quantitative understanding.

The second characteristic energy corresponds to the spin splitting needed to destabilize singlet $\mathrm{X}^{+}$. This hardly depends on the surrounding hole gas since we observe that the spin splitting at the destabilization point remains between 2 and $3 \mathrm{meV}$, regardless of the hole densities studied here [33].

Finally, the energy left in the system after the $D_{\text {low }}$ transition, experimentally determined by the energy difference between $D_{\text {low }}$ and $D_{\text {high }}$, is plotted by full symbols in Fig. 6. The excited states should be discussed in terms of a combination of single particle excitations, plasmons, many-body excitations, with a total wave vector equal to $2 k_{\mathrm{F}}$ ( $k_{\mathrm{F}}$ being the wave vector at the Fermi energy of the hole gas in zero magnetic field). The $D_{\text {low }}-D_{\text {high }}$ splitting tends to fit the Fermi energy at large carrier density, as expected for the single band-to-band transition described in Sec. 4.1.2.

\section{Conclusion}

In conclusion, we have presented an analysis of PL spectra of $p$-type modulation doped (Cd,Mn)Te quantum wells at various spin splittings and hole densities up to $5 \times 10^{11} \mathrm{~cm}^{-2}$. At small spin splitting, $\mathrm{X}^{+}$is formed in its singlet state whatever the hole density. When the hole spin splitting exceeds about $3 \mathrm{meV}$ and regardless of the hole density, the PL exhibits a double line $\left(D_{\text {low }}\right.$ and $\left.D_{\text {high }}\right)$ in the presence of carriers, and a single line $(\mathrm{X})$ at vanishing carrier density. The destabilization of $\mathrm{X}^{+}$is ascribed to a crossing of its state with another state where all holes have the same spin orientation. The double line is attributed to band-to-band transitions: the high energy component $D_{\text {high }}$ leads, through an indirect transition in $k$-space, to the ground state of the system, whereas $D_{\text {low }}$ leads to an excited state of the hole gas. The spin splitting needed to fully polarize the hole gas is found to be around twice smaller than the expected Fermi energy of the gas (calculated from the hole density and effective masses). Furthermore, the splitting between the two components $\left(D_{\text {low }}\right.$ and $D_{\text {high }}$ ) of the double line does not match the Fermi energy of the hole gas below a carrier density of about $2 \times 10^{11} \mathrm{~cm}^{-2}$. Therefore the single particle image used here made it possible to draw the evolution of the different states involved in the PL processes and to access the typical energies of the system. However, further theoretical work - treating hole-hole interactions — should be done to achieve a quantitative understanding. 


\section{References}

[1] M. Lampert, Phys. Rev. Lett. 1, 450 (1958).

[2] K. Kheng, R.T. Cox, Y. Merle d'Aubigné, Franck Bassani, K. Saminadayar, S. Tatarenko, Phys. Rev. Lett. 71, 1752 (1993).

[3] G.V. Astakhov, D.R. Yakovlev, V.P. Kochereshko, W. Ossau, W. Faschinger, J. Puls, F. Hanneberger, S.A. Crooker, Q. McCulloch, D. Wolverson, N.A. Gippius, A. Waag, Phys. Rev. B 65, 165335 (2002).

[4] A. Esser, E. Runge, R. Zimmermann, W. Langbein, Phys. Rev. B 62, 8232 (2000).

[5] P. Redlinski, J. Kossut, Solid State Commun. 118, 295 (2001).

[6] C. Riva, F.M. Peeters, K. Varga, Phys. Rev. B 61, 13873 (2000).

[7] B. Stébé, E. Feddi, A. Ainane, F. Dujardin, Phys. Rev. B 58, 9926 (1998).

[8] G.V. Astakhov, V.P. Kochereshko, D.R. Yakovlev, W. Ossau, J. Nurnberger, W. Faschinger, G. Landwehr, T. Wojtowicz, G. Karczewski, J. Kossut, Phys. Rev. B 65, 115310 (2002).

[9] K. Kheng, Ann. Phys. 20, C2-229 (1995).

[10] S. Lovisa, R.T. Cox, N. Magnea, K. Saminadayar, Phys. Rev. B 56, R12787 (1997).

[11] G. Finkelstein, V. Umansky, I. Bar-Joseph, V. Ciulin, S. Haacke, J.-D. Ganiere, B. Deveaud, Phys. Rev. B 58, 12367 (1998).

[12] V. Ciulin, P. Kossacki, S. Haacke, J.-D. Ganiere, B. Deveaud, A. Esser, M. Kutrowski, T. Wojtowicz, Phys. Rev. B 62, R16310 (2000).

[13] H.W. Yoon, A. Ron, M.D. Sturge, L.N. Pfeirer, Solid State Commun. 100, 743 (1996).

[14] P. Kossacki, V. Ciulin, M. Kutrowski, J.-D. Ganiere, T. Wojtowicz, B. Deveaud, Phys. Status Solidi B 229, 659 (2002).

[15] M. Kutrowski, T. Wojtowicz, P. Kossacki, V. Ciulin, J. Kossut, Phys. Status Solidi B 229, 791 (2002).

[16] V. Ciulin, P. Kossacki, M. Kutrowski, J.-D. Ganiere, T. Wojtowicz, B. Deveaud, Phys. Status Solidi B 229, 627 (2002).

[17] S.A. Crooker, E. Johnston-Halperin, D.D. Awschalom, R. Knobel, N. Samarth, Phys. Rev. B 61, R16307 (2000).

[18] D. Sanvitto, D.M. Whittaker, A.J. Shields, M.Y. Simmons, D.A. Ritchie, M. Pepper, Phys. Rev. Lett. 89, 246805 (2002).

[19] A.J. Shields, M. Pepper, M.Y. Simmons, D.A. Ritchie, Phys. Rev. B 52, 841 (1995).

[20] O. Homburg, K. Sebald, P. Michler, J. Gutowski, H. Wenisch, D. Hommel, Phys. Rev. B 62, 7413 (2000).

[21] C. Riva, F.M. Peeters, K. Varga, Phys. Rev. B 63, 115302 (2001).

[22] B. Cole, T. Takamasu, K. Takehana, R. Goldhahn, D. Schulze, G. Kido, J.M. Chamberlain, G. Gobsch, M. Hemini, G. Hill, Physica B 249-251, 607 (1998). 
[23] V. Huard, R. Cox, K. Saminadayar, A. Arnoult, S. Tatarenko, Phys. Rev. Lett. 84, 187 (2000).

[24] P. Kossacki, J. Cibert, D. Ferrand, Y. Merle d'Aubigné, A. Arnoult, A. Wasiela, S. Tatarenko, J. Gaj, Phys. Rev. B 60, 16018 (1999).

[25] V. Huard, R. Cox, K. Saminadayar, C. Bourgognon, A. Arnoult, J. Cibert, S. Tatarenko, M. Potemski, Phys. Status Solidi A 178, 95 (2000).

[26] T. Wojtowicz, M. Kutrowski, G. Karczewski, J. Kossut, Appl. Phys. Lett. 73, 1379 (1998).

[27] A. Esser, R. Zimmermann, E. Runge, Phys. Status Solidi B 227, 317 (2001).

[28] R.A. Suris, V.P. Kochereshko, G.V. Astakhov, D.R. Yakovlev, W. Ossau, J. Nurnberger, W. Faschinger, G. Landwehr, T. Wojtowicz, G. Karczewski, J. Kossut, Phys. Status Solidi B 227, 343 (2001).

[29] W. Maslana, M. Bertolini, H. Boukari, P. Kossacki, D. Ferrand, J.A. Gaj, S. Tatarenko, J. Cibert, Appl. Phys. Lett. 82, 1875 (2003).

[30] A.J. Shields, J.L. Osborne, M.Y. Simmons, M. Pepper, D.A. Ritchie, Phys. Rev. B 52, R5523 (1995).

[31] A.J. Shields, J.L. Osborne, M.Y. Simmons, D.A. Ritchie, M. Pepper, Semicond. Sci. Technol. 11, 890 (1996).

[32] H. Boukari, P. Kossacki, M. Bertolini, D. Ferrand, J. Cibert, S. Tatarenko, A. Wasiela, J.A. Gaj, T. Dietl, Phys. Rev. Lett. 88, 207204 (2002).

[33] P. Kossacki, H. Boukari, M. Bertolini, D. Ferrand, J. Cibert, S. Tatarenko, J.A. Gaj, B. Deveaud, V. Ciulin, M. Potemski, http://xxx.lanl.gov/archive/condmat/0404490 (2004).

[34] R. Planel, J. Gaj, C. Benoit, J. Phys. (France) C 41, C5-39 (1980).

[35] P. Kossacki, V. Ciulin, M. Kutrowski, J.-D. Ganiere, T. Wojtowicz, B. Deveaud, in: Proc. Int. Conf. on Physics of Semiconductors, Osaka 2000, Eds. N. Miura, T. Ando, Springer, Berlin 2001, p. 623.

[36] Le Si Dang, G. Neu, R. Romestain, Solid State Commun. 44, 1187 (1982).

[37] G. Fishman, Phys. Rev. B 52, 11132 (1995).

[38] T. Jungwirth, B. Lee, A.H. MacDonald, Physica E 10, 153 (2001).

[39] T. Dietl, A. Haury, Y. Merle d'Aubigné, Phys. Rev. B 55, 3347 (1997).

[40] T. Ando, A.B. Fowler, F. Stern, Rev. Mod. Phys. 54, 437 (1982).

[41] J. Zhu, H.L. Stormer, L.N. Pfeiffer, K.W. Baldwin, K.W. West, Phys. Rev. Lett. 90, 056805 (2003). 